\title{
Application of VLSI In Artificial Intelligence
}

\author{
M. Zamin Ali Khan ${ }^{1}$, Hussain Saleem ${ }^{2}$ and Shiraz Afzal ${ }^{3}$ \\ ${ }^{1}$ Main Communication Network Department, University of Karachi, Karachi, Pakistan \\ ${ }^{2}$ Department of Computer Science, University of Karachi, Karachi, Pakistan \\ ${ }^{3}$ Electronic Engineering Departments, Sir Syed University of Engineering \& Technology, Karachi, Pakistan
}

\begin{abstract}
This paper describes the application of VLSI (Very Large Scale Integrated) circuit in Artificial Intelligence (AI). VLSI is the inter-disciplinary science of utilizing advance semiconductor technology to create various functions of computer system. This manuscript addresses the close link of microelectronics and AI. By combining VLSI technology, a very powerful computer architecture confinement is possible.
\end{abstract}

Keywords - VLSI, Artificial Intelligence, DAA, Chip Design, Expert System.

\section{INTRODUCTION}

Gone are the days when massive computers made-up of vacuum tubes sat humming in entire dedicated rooms and could do about 360 multiplications of 10 digit numbers in a second. Though they were heralded as the fastest computing machines of that time, they surely do not stand a chance when compared to the modern day machines. Modern day computers are getting smaller, faster, and in-expensive and more power and efficient every progressing second. But what drove this change? The whole domain of computing ushered into a new dawn of electronic miniaturization with the advent of semiconductor transistor by Bardeen (1947-48) and then the Bipolar Transistor by Shockley (1949) in the Bell Laboratory [1].

The development of microelectronics spans a time which is even lesser than the average life expectancy of a human, and yet it has seen as many as four generations. Early 60's saw the low density fabrication processes classified under Small Scale Integration (SSI) in which transistor count was limited to about 10. This rapidly gave way to Medium Scale Integration (MSI) in the late 60's when around 100 transistors could be placed on a single chip.

It was the period when the cost of research began to deterioration and private firms started entering the competition in comparison to the former years where the main load was borne by the military. TransistorTransistor logic (TTL) proposing higher integration densities endured other IC families identically EmitterCoupled Logic (ECL) and became the basis of the first integrated circuit revolution. It was the creation of this family that gave inspiration to semiconductor giants for example Texas Instruments, Fairchild, Motorola and National Semiconductors. Early seventies marked the progression of transistor count to about 1000 per chip called the Large Scale Integration (LSI).

By mid-eighties, the transistor count on a single chip had already exceeded 1000 and henceforth came the era of Very Large Scale Integration (VLSI). Though numerous improvements have been made and the transistor count is still increasing, further the names of generations e.g. ULSI (Ultra Large Scale Integration) are normally avoided. It was during this time when TTL got defeated in the battle to MOS (Metal Oxide Semiconductor) family due to the same problems that had pushed vacuum tubes into slackness, power dissipation and the limit it imposed on the number of gates that could be positioned on a single die [1].

The second age of Integrated Circuits revolution started with the starter of the first microprocessor, the 4004 by Intel in 1972 and the 8080 in 1974. Today various companies like Texas Instruments, Infineon, Alliance Semiconductors, Cadence, Synopsys, Cisco, Micron Tech, National Semiconductors, ST Microelectronics, Qualcomm, Lucent, Mentor Graphics, Analog Devices, Intel, Philips, Motorola and many other firms have been proven and are dedicated to many fields in VLSI like Programmable Logic Devices, Hardware Descriptive Languages, Design tools, Embedded Systems etc.

With the Introduction in Section-1, Section-2 describes the notion of Artificial Intelligence. Section-3 gives comparison of AI \& VLSI. At the end, Section-4 gives detail about the importance and applications of VLSI in AI.

\section{ARTIFICIAL INTELLIGENCE}

AI is the area of computer science focusing on creating machines that can engage on behaviors that humans consider intelligent. The ability to create intelligent machines has intrigued humans since ancient times and today with the advent of the computer and 50 years of research into AI programming techniques, the dream of smart machines is becoming a reality. Researchers are creating systems which can mimic human thought, understand speech, beat the best human chess player, and countless other feats that were never possible before [2]. 


\section{ARTIFICIAL INTELLIGENCE \& VLSI (AI AND VLSI)}

Most of the prosperous applications of Artificial Intelligence (AI) to integrated circuit and printed circuit board design schematic have appeared in the area of expert systems which work as design assistants [3][9]. But expert systems technology is only one aspect of AI. VLSI design is a complex process. Moreover, that complexity is multi-dimensional. Size and level of detail are two distinctive dimensions. Others include the hierarchical nature of the design process as well as of the self-design. AI languages lend themselves to solving problems of such complexity. These language features coupled with expert systems permit a significant first step to solving a very difficult problem i.e. the verification of the correctness of a design [3].

\section{APPLICATIONS}

Applications of AI are growing rapidly. These are being pursued not only in university research but also in industrial environments. One such application area under investigation is VLSI design [7][8][11]. The first significant applications were expert systems. An expert system is essentially a computer program which simulates a human expert's behavior by applying reasoning techniques to a base of knowledge in some area of specialization. An expert system should be able to solve straight forward and medium difficulty problems in that area of specialization. For very difficult problems, it should be able to formulate one or more potential solutions and possibly indicate the reliability of each alternative.

An expert system differs from a standard computer program in several important aspects. In a conventional computer program, the "intelligence" is directly written into the code. The code then solves a problem by performing an algorithm which is known to address the problem. Further, except for explicit branches, the code executes sequentially. In an expert system, the "intelligence" is separated from the control or reasoning portion. Thus changes and additions to the knowledge can occur without changing the control portion [4].

The essence of the Knowledge based approach used in AI, is to ask what knowledge is used by a human expert in solving some task and to develop algorithms and data structures that may represent this knowledge explicitly. Often the most useful facts are a collection of some rules of thumb, derived through subjective approach, can be stated as IF-THEN rules and can be used in a simple reasoning process. Researchers using this reasoning approach in a number of areas of VLSI have found several advantages over the trivial approaches, such as discussed in [4].

- It is easier to make incremental improvements

- It is easier for the system to explain what it is doing and why

- It is easier for the human expert to determine what is incorrect or incomplete about the systems knowledge and explain how to fix it

- It is easier to interactively use a human expert's abilities

These experts system are being used widely in VLSI design [7][8][10][12]. The Design Automation Assistant (DAA) [5] is important as it was one of the first knowledge-based systems created for VLSI design. It was developed jointly by researchers at Carnegie-Mellon University and AT\&T Bell Labs. The initial DAA contained rules describing various synthesis operations and applicable contexts. Production rules were expressed in terms of registers, operators, data paths, and control signals. Over the years, the DAA system has been expanded and refined [3]. There are more than 500 rules in its knowledge base. It has successfully been used to design several systems.

Another important example is the Design Advisor by NCR [3] which is an expert assistant offering advice in six areas for the design of semi-custom CMOS chips from a library of standard cells. The advisor includes facts and rules on simulation, functions, timing, testability, design rules, and specification feasibility. The Design Advisor is built on top of an expert system shell called Proteus, developed by the Microelectronics and Computer Technology Corporation (MCC).

Another knowledge based system called REDESIGN [4] is being used by several people to address the following problem of functional redesign. Given an existing circuit, its functional specifications, and a desired change to these specifications. It helps the user to determine a change in the circuit that will allow it to meet the altered functional specifications without introducing undesirable side-effects. In this case, providing intelligent assistance on two quite different types of reasoning about the given circuit is desired.

The first essential type of reasoning about circuits is causal reasoning about the inter-relations among signals within the circuit. This is a generalization of the notions of circuit simulation and symbolic simulation. It involves tasks such as, given a description of the streams of data being input to a component, deriving a description of the output data streams. Or, given a specification of the required characteristics of the outputs of a component, determine what characteristics must be satisfied by the inputs to the component. The subsystem of REDESIGN which has been developed to solve these kinds of problems is called CRITTER [6]. A second type of reasoning essential to redesign involves reasoning about purposes of components. For example, given a circuit, and its specifications, explain the role played by a particular component in implementing the overall 
specifications. Or, determine the range of components that can be substituted for this component without violating the overall specifications.

\section{REFERENCES}

[1] Engineers Garage, Web: http://www.engineersgarage.com/articles/vlsi-design-future, Accessed on: 30 September 2012.

[2] Oracle ThinkQuest, Web: http://library.thinkquest.org/2705/, Accessed on: 30 September 2012.

[3] Ann Miller, “From Expert Assistant to Design Verification: Applications of AI to VLSI Design", Session 11Al, IEEE, Vol.2, pp.406410, 1989.

[4] Louis I. Steinberg, Tom M. Mitchell, “A knowledge based approach to VLSI CAD the redesign system”, In Proceedings of the 21st Design Automation Conference, IEEE Press, Piscataway, NJ, USA, pp.412-418, 1984.

[5] T. J. Kowalski, D.E. Thomas, "The VLSI Design Automation Assistant Prototype System", In 20th Design Automation Conference, IEEE, pp.479-483, 1983.

[6] Van E. Kelly, "The CRITTER System: Automated Critiquing of Digital Circuit Designs", In Proceedings of the 21st Design Automation Conference, IEEE Press, 1984, 419-425.

[7] R. L. Steele, “An Expert System Application in Semicustom VLSI Design", In Proceedings of the 24th ACM/IEEE Design Automation Conference, A. O'Neill and D. Thomas (Eds.), ACM, pp.679-688, 1987.

[8] H. Farahat, A. Eldessouki, M. Y. Mahmoud, H. Elsimary, "An Expert System for VLSI Layout Design", International Conference on Systems Engineering, IEEE, pp.529-532, 1989.

[9] M. Stefik, L. Conway, "Towards the Principled Engineering of Knowledge", AI Magazine, Vol. 3:3, pp.4-16, 1982. (Reprinted in Readings From the AI Magazine, Volumes 1-5, 1980-1985, pp.135-147, 1988.)

[10] J. Kim, J.P. McDermott, "TALIB: An IC Layout Design Assistant”, In Proc. AAAI, pp.197-201, 1983.

[11] Guy Rabbat, "VLSI and AI are getting closer", Circuits and Devices Magazine, IEEE, Vol.4, No.1, pp.15-18, 1988.

[12] T.J. Kowalski, D. J. Geiger, W.H. Wolf, W. Fichtner, "The VLSI Design Automation Assistant: From Algorithms to Silicon", Design \& Test of Computers, IEEE, Vol.2, No.4, pp.33-43, 1985.

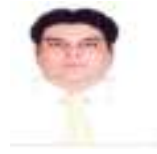

Dr. M. Zamin Ali Khan is a Head of Main Communication network department at University of Karachi. He has received B.E (Electrical Engineering) from NED University, Karachi, Pakistan and MS (Electrical and Computer Engineering) from Concordia University, Montreal, Canada and PhD in Computer Science from UoK. He has more than 18 years of experience of teaching and industry. He has worked in Victhom Human Bionics, Canada as an Engineer Scientist. Currently, he is a senior member of Pakistan Engineering Council, Canadian Engineering Council and IEEE. His research interest includes VLSI, Digital design, Digital signal processing and Analog front end of wireless devices.

Hussain Saleem is currently Assistant Professor and Ph.D. Research Scholar at Department of Computer Science, University of Karachi, Pakistan. He received B.S. in Electronics Engineering from Sir Syed University of Engineering \& Technology, Karachi in 1997 and has done Masters in Computer Science from University of Karachi in 2001. He bears vast experience of about 15 years of University Teaching, Administration and Research in various dimensions of Computer Science. Hussain is the Author of several International Journal publications. His field of interest is Software Science, System Automation, Hardware design and engineering, and Simulation \& Modeling. He is senior member of Pakistan Engineering Council (PEC).

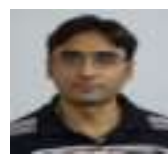

Shiraz Afzal is a full time faculty member at Sir Syed University of Engineering \& Technology, Karachi, Pakistan. He received his B.S. in Electronics from Sir Syed University of Engineering and Technology Karachi, Pakistan and M.E degree in Electronics with specialization in Micro-System design from NED University of Engineering and Technology Karachi, Pakistan in 2006 and 2012 respectively. His research interest includes Microelectronic circuit design. He is also a member of PEC. 